\title{
A REMARK ON UNIQUENESS FOR QUASILINEAR ELLIPTIC EQUATIONS
}

\author{
N. ANDRÉ and M. CHIPOT \\ Département de Mathématiques, Université de Metz \\ Ile du Saulcy, 57045 Metz Cedex 01, France
}

1. Introduction. Let $\Omega$ be a bounded open subset of $\mathbf{R}^{n}, n \geq 1$. Assume that $a(x, u)$ is a Carathéodory function satisfying

$$
0<\alpha \leq a(x, u) \leq \beta \quad \text { a.e. } x \in \Omega, \quad \forall u \in \mathbf{R}
$$

where $\alpha, \beta$ are two positive constants. For $f \in H^{-1}(\Omega), g \in H^{1}(\Omega)$ we would like to consider here the problem

$$
\left\{\begin{array}{l}
-\frac{\partial}{\partial x_{i}}\left(a(x, u) \frac{\partial u}{\partial x_{i}}\right)=f \quad \text { in } \Omega, \\
u-g \in H_{0}^{1}(\Omega) .
\end{array}\right.
$$

We use the summation convention and we refer to $[\mathrm{GT}]$ or $[\mathrm{KS}]$ for the definition of the Sobolev spaces used throughout the paper.

First, under the above assumptions, using a fixed point argument of Schauder type, it is very easy to show that (1.2) admits a solution (see for instance [CM]). We would like to investigate here the question of uniqueness. More precisely we would like to prove the following result:

Theorem 1. Assume that for some positive constant $C$ one has

$$
|a(x, u)-a(y, u)| \leq C|x-y| \quad \forall u \in \mathbf{R}, \forall x, y \in \Omega
$$

or

$$
|a(x, u)-a(x, v)| \leq C|u-v| \quad \forall u, v \in \mathbf{R}, \text { a.e. } x \in \Omega
$$

then the problem (1.2) has a unique solution $(||$ denotes the usual euclidean norm in $\left.\mathbf{R}^{p}\right)$. If (1.3), (1.4) fail then uniqueness can fail even if $u \rightarrow a(x, u)$ is Hölder continuous of any order.

1991 Mathematics Subject Classification: Primary 35J65.

The paper is in final form and no version of it will be published elsewhere. 
Remark 1.1. Loosely speaking uniqueness holds if and only if either $\left|\nabla_{x} a(x, u)\right|$ or $\partial a(x, u) / \partial u$ are uniformly bounded. In fact, it has been pointed out to us by P. Bénilan that $\nabla_{x} a(x, u) \in L^{2}(\Omega)$ is enough to insure here uniqueness.

This kind of problems were considered before by several authors (see $[\mathrm{CC}],[\mathrm{CM}],[\mathrm{M}]$, $[\mathrm{T}]$ ), however, even in this simple case the picture was not yet complete. In particular, no counterexample seems to be known.

2. The proof of uniqueness. Let us first consider the case where (1.3) holds. Then set

$$
A(x, s)=\int_{0}^{s} a(x, t) d t
$$

If $u \in H^{1}(\Omega)$ then it is clear that

$$
A(x, u(x)) \in H^{1}(\Omega) .
$$

Moreover, in the distributional sense one has

$$
\frac{\partial}{\partial x_{i}} A(x, u)=a(x, u) \frac{\partial u}{\partial x_{i}}+\int_{0}^{u(x)} \frac{\partial a(x, t)}{\partial x_{i}} d t .
$$

Then let us prove:

Proposition 2.1. Assume that (1.3) holds. Then (1.2) has a unique solution.

Proof. Let us denote by $u, v$ two solutions of (1.1). By subtraction we get

$$
-\frac{\partial}{\partial x_{i}}\left(a(x, u) \frac{\partial u}{\partial x_{i}}-a(x, v) \frac{\partial v}{\partial x_{i}}\right)=0 \quad \text { in } \Omega .
$$

But thanks to (2.3) this reads also

$$
-\frac{\partial}{\partial x_{i}}\left(\frac{\partial}{\partial x_{i}}(A(x, u)-A(x, v))-\int_{v(x)}^{u(x)} \frac{\partial a(x, t)}{\partial x_{i}} d t\right)=0 \quad \text { in } \Omega .
$$

If $f$ is a function we denote by $[f>0]$ the set defined by $[f>0]=\{x \in \Omega \mid f(x)>0\}$ and we use similar notation for $[0<f \leq \epsilon]$. Then we have

LEMma 2.1. For any $\xi \in C^{1}(\bar{\Omega})$

$$
\int_{[u-v>0]}\left[\frac{\partial}{\partial x_{i}}(A(x, u)-A(x, v))-\int_{v(x)}^{u(x)} \frac{\partial a(x, t)}{\partial x_{i}} d t\right] \frac{\partial \xi}{\partial x_{i}} d x=0 .
$$

Let us postpone for the time being the proof of this lemma. Then, integrating by parts in (2.6) we obtain (recall that $A(x, u)-A(x, v) \in H_{0}^{1}(\Omega)$ )

$$
\int_{[u-v>0]} A(x, u)-A(x, v) \frac{\partial^{2} \xi}{\partial x_{i}^{2}}+\int_{v(x)}^{u(x)} \frac{\partial a(x, t)}{\partial x_{i}} d t \frac{\partial \xi}{\partial x_{i}} d x
$$

which reads also

$$
\int_{[u-v>0]} \int_{v(x)}^{u(x)}\left[a(x, t) \frac{\partial^{2} \xi}{\partial x_{i}^{2}}+\frac{\partial a(x, t)}{\partial x_{i}} \frac{\partial \xi}{\partial x_{i}}\right] d t d x=0
$$


Then, choosing $\xi=e^{\gamma x_{1}}$ in (2.7), we have for $\gamma$ large enough and by (1.3)

$$
a(x, t) \frac{\partial^{2} \xi}{\partial x_{i}^{2}}+\frac{\partial a(x, t)}{\partial x_{i}} \frac{\partial \xi}{\partial x_{i}} \geq e^{\gamma x_{1}}\left(\gamma^{2} \alpha-C \gamma\right)>0
$$

and then in order for (2.7) to hold, $[u-v>0]$ must have measure 0 . This leads to $u \leq v$ and reversing the role of $u$ and $v$ to $u=v$.

Proof of the lemma. Let us denote by ()$^{+}$the positive part of a function, by $\min [$,$] the minimum of two functions. Remark then that for \xi \in C^{1}(\bar{\Omega}), \epsilon>0$,

$$
\min \left[(A(x, u)-A(x, v))^{+} / \epsilon, 1\right] \cdot \xi \in H_{0}^{1}(\Omega) .
$$

Thus, multiplying (2.5) by the above function and integrating over $\Omega$ we deduce (for simplicity we set below $A(x, u)=A(u))$,

$$
\begin{aligned}
& \int_{\Omega}\left[\frac{\partial}{\partial x_{i}}(A(u)-A(v))-\int_{v(x)}^{u(x)} \frac{\partial a(x, t)}{\partial x_{i}} d t\right] \min \left[(A(u)-A(v))^{+} / \epsilon\right] \frac{\partial \xi}{\partial x_{i}} d x \\
= & -\int_{[0<A(u)-A(v) \leq \epsilon]}\left[\frac{\partial}{\partial x_{i}}(A(u)-A(v))-\int_{v(x)}^{u(x)} \frac{\partial a(x, t)}{\partial x_{i}} d t\right] \frac{\partial}{\partial x_{i}}\left(\frac{A(u)-A(v)}{\epsilon}\right) \xi d x .
\end{aligned}
$$

Let us denote by $\chi_{A}$ the characteristic function of the set $A$. By (1.1) one has

$$
[0<u-v]=[0<A(u)-A(v)] .
$$

So, when $\epsilon \rightarrow 0$

$$
\min \left[(A(u)-A(v))^{+} / \epsilon, 1\right] \rightarrow \chi_{[0<u-v]} \quad \text { a.e. in } \Omega .
$$

It follows, by the Lebesgue convergence theorem, that the first integral in (2.9) converges to

$$
\int_{[0<u-v]}\left[\frac{\partial}{\partial x_{i}}(A(u)-A(v))-\int_{v(x)}^{u(x)} \frac{\partial a(x, t)}{\partial x_{i}} d t\right] \frac{\partial \xi}{\partial x_{i}} d x .
$$

Now, we claim that for $\xi \geq 0$

$$
\lim _{\epsilon \rightarrow 0} \int_{[0<A(u)-A(v) \leq \epsilon]}\left[\frac{\partial}{\partial x_{i}}(A(u)-A(v))-\int_{v(x)}^{u(x)} \frac{\partial a(x, t)}{\partial x_{i}} d t\right] \frac{\partial}{\partial x_{i}}\left(\frac{A(u)-A(v)}{\epsilon}\right) \xi d x \geq 0 .
$$

Indeed, this integral reads also

$$
\begin{aligned}
& \frac{1}{\epsilon} \int_{[0<A(u)-A(v) \leq \epsilon]}|\nabla(A(u)-A(v))|^{2} \xi d x \\
&-\int_{[0<A(u)-A(v) \leq \epsilon]} \int_{v(x)}^{u(x)} \frac{\partial a(x, t)}{\partial x_{i}} d t \frac{\partial}{\partial x_{i}}\left(\frac{A(u)-A(v)}{\epsilon}\right) \xi d x \\
& \geq-\int_{[0<A(u)-A(v) \leq \epsilon] v(x)}^{u(x)} \frac{\partial a(x, t)}{\partial x_{i}} d t \frac{\partial}{\partial x_{i}}\left(\frac{A(u)-A(v)}{\epsilon}\right) \xi d x
\end{aligned}
$$




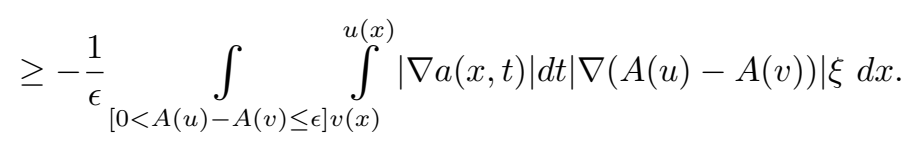

Now, by (1.3) one has for some constant $C$

$$
\int_{v(x)}^{u(x)}|\nabla a(x, t)| d t \leq C(u(x)-v(x)) .
$$

Moreover, when $u \geq v$ one has

$$
\alpha(u(x)-v(x)) \leq \int_{v(x)}^{u(x)} a(x, t) d t=A(u)-A(v) .
$$

Hence on $[0<A(u)-A(v) \leq \epsilon]$

$$
\int_{v(x)}^{u(x)}|\nabla a(x, t)| d t \leq \frac{C}{\alpha}(A(u)-A(v)) \leq \frac{C}{\alpha} \epsilon
$$

and the last integral in (2.10) is bounded from below by

$$
-\frac{C}{\alpha} \underset{[0<A(u)-A(v) \leq \epsilon]}{\int}|\nabla(A(u)-A(v))| \xi d x
$$

which goes to 0 with $\epsilon$.

Collecting the above results and letting $\epsilon \rightarrow 0$ in $(2.9)$ we obtain for $\xi \in C^{1}(\bar{\Omega}), \xi \geq 0$

$$
\int_{[u-v>0]}\left[\frac{\partial}{\partial x_{i}}(A(x, u)-A(x, v))-\int_{v(x)}^{u(x)} \frac{\partial a(x, t)}{\partial x_{i}} d t\right] \frac{\partial \xi}{\partial x_{i}} d x \leq 0 .
$$

Changing $\xi$ into $M-\xi$ for $M$ large enough such that $M-\xi \geq 0$ leads to (2.6) for any $\xi \in C^{1}(\bar{\Omega})$.

Let us now turn to the case where (1.4) holds. In fact, we would like to use here a slightly more general assumption. Indeed let us set

$$
\omega_{a}(t)=\sup _{x \in \Omega,|u-v| \leq t}|a(x, u)-a(x, v)|
$$

and let us assume

$$
\int_{0^{+}} \frac{d s}{\omega_{a}(s)}=+\infty .
$$

Clearly if (1.4) holds one has $\omega_{a}(t) \leq C t$ and (2.12) holds.

Then we have:

Proposition 2.2. Assume that (2.11), (2.12) hold. Then (1.2) has a unique solution.

Proof. Let us denote again by $u, v$ two solutions of (1.1). Then for $\epsilon>0$ let us set

$$
F_{\epsilon}= \begin{cases}\int_{\epsilon}^{x} d s / \omega(s)^{2} & \text { when } x \geq \epsilon, \\ 0 & \text { when } x<\epsilon,\end{cases}
$$


where for the sake of simplicity we have set $\omega_{a}=\omega$ (note that $\omega(t)>0$ when $t>0$ unless $a$ is independent of $u$ ). From (2.4), multiplying by

$$
F_{\epsilon}(u-v) \in H_{0}^{1}(\Omega)
$$

and integrating by parts, we get

$$
\int_{\Omega}\left(a(x, u) \frac{\partial u}{\partial x_{i}}-a(x, v) \frac{\partial v}{\partial x_{i}}\right) \cdot \frac{\partial}{\partial x_{i}} F_{\epsilon}(u-v) d x=0 .
$$

This can be rewritten as

$$
\begin{aligned}
\int_{\Omega} a(x, u) \frac{\partial(u-v)}{\partial x_{i}} \cdot \frac{\partial}{\partial x_{i}} F_{\epsilon} & (u-v) d x \\
= & -\int_{\Omega}(a(x, u)-a(x, v)) \frac{\partial v}{\partial x_{i}} \cdot \frac{\partial}{\partial x_{i}} F_{\epsilon}(u-v) d x .
\end{aligned}
$$

From (2.13) we deduce

$$
\begin{aligned}
\int_{[u-v>\epsilon]} a(x, u) \frac{|\nabla(u-v)|^{2}}{\omega^{2}(u-v)} & d x \\
& =-\int_{[u-v>\epsilon]} \frac{(a(x, u)-a(x, v))}{\omega^{2}(u-v)} \frac{\partial v}{\partial x_{i}} \cdot \frac{\partial}{\partial x_{i}}(u-v) d x \\
& \leq \int_{[u-v>\epsilon]} \frac{\omega(u-v)}{\omega^{2}(u-v)}|\nabla v||\nabla(u-v)| d x \\
& =\int_{[u-v>\epsilon]}|\nabla v| \frac{|\nabla(u-v)|}{\omega(u-v)} d x .
\end{aligned}
$$

Hence by (1.1) and the Cauchy-Schwarz inequality we obtain

$$
\alpha \int_{[u-v>\epsilon]} \frac{|\nabla(u-v)|^{2}}{\omega^{2}(u-v)} d x \leq\left[\int_{[u-v>\epsilon]}|\nabla v|^{2} d x\right]^{\frac{1}{2}}\left[\int_{[u-v>\epsilon]} \frac{|\nabla(u-v)|^{2}}{\omega^{2}(u-v)} d x\right]^{\frac{1}{2}}
$$

from which it follows

$$
\int_{[u-v>\epsilon]} \frac{|\nabla(u-v)|^{2}}{\omega^{2}(u-v)} d x \leq \frac{1}{\alpha^{2}} \int_{\Omega}|\nabla v|^{2} d x
$$

Set

Then the above inequality reads

$$
G_{\epsilon}= \begin{cases}\int_{\epsilon}^{x} \frac{d s}{\omega(s)} & \text { when } x \geq \epsilon, \\ 0 & \text { when } x<\epsilon .\end{cases}
$$

$$
\int_{\Omega}\left|\nabla G_{\epsilon}(u-v)\right|^{2} d x \leq \frac{1}{\alpha} \int_{\Omega}|\nabla v|^{2} d x .
$$

Hence, by the Poincaré Inequality (see [BKS] for a similar argument), for some positive 
constant $C$

$$
\int_{\Omega}\left|G_{\epsilon}(u-v)\right|^{2} d x \leq C \int_{\Omega}|\nabla v|^{2} d x .
$$

Letting $\epsilon$ go to 0 we deduce by (2.12) that $u \leq v$ and the result follows by exchanging the role of $u$ and $v$.

So, we have established the part of Theorem 1 regarding uniqueness. Let us turn now to the second part of this theorem.

3. A class of counterexamples. We are going to construct one dimensional counterexamples. So, for $\Omega=\left(a_{1}, a_{2}\right)$ we will consider the problem

$$
\left\{\begin{array}{l}
-\left(a(x, u) u^{\prime}\right)^{\prime}=f \quad \text { in } \Omega, \\
u\left(a_{1}\right)=A_{1}, \quad u\left(a_{2}\right)=A_{2},
\end{array}\right.
$$

where $a_{1}, a_{2}, A_{1}, A_{2}$ are constants. Let us prove:

Proposition 3.1. Assume that (1.3), or (2.11), (2.12) fail, then (1.2) or (3.1) may have several solutions even if $u \rightarrow a(x, u)$ is Hölder continuous of any order $\gamma, \gamma \in(0,1)$ i.e. even if

$$
|a(x, u)-a(x, v)| \leq C|u-v|^{\gamma} \quad \forall u, v \in \mathbf{R}, \text { a.e. } x \in \Omega \text {. }
$$

Pr o of. Let $\omega$ be a nondecreasing, continuous function such that

$$
\omega(0)=0, \quad \omega(t)>0 \quad \forall t>0, \quad \int_{0^{+}} \frac{d s}{\omega(s)}<+\infty,
$$

$\omega(t) / t$ is nonincreasing.

We are going to construct a counterexample to uniqueness of the type of (3.1) with an $a$ having a modulus of continuity $\omega_{a}$ equivalent to $\omega$. Set

$$
\theta(s)=\int_{0}^{s} \frac{d s}{\omega(s)}
$$

Then, $\theta$ is a one-to-one mapping from $[0, T]$ into $[0, \theta(T)]$ for any $T>0$. Let us denote by $\theta^{-1}$ its inverse. One has clearly

$$
\frac{d \theta^{-1}}{d y}(y)=\omega\left(\theta^{-1}(y)\right) \quad \forall y>0 .
$$

Let $u$ be a smooth increasing function defined on $\left(a_{1}, a_{2}\right)$, and such that $u\left(a_{1}\right)=A_{1}<$ $A_{2}=u\left(a_{2}\right)$. Then, let us define $v$ by

$$
v= \begin{cases}u+\theta^{-1}\left(x-a_{1}\right) & \text { in a neighbourhood of } a_{1}, \\ u+\theta^{-1}\left(a_{2}-x\right) & \text { in a neighbourhood of } a_{2},\end{cases}
$$

$v$ being smooth and such that

$$
v>u, \quad v^{\prime}>0 \quad \text { on }\left(a_{1}, a_{2}\right) .
$$


It is clear that such a definition is always possible. Now, let us define $a(x, u)$ by setting

$$
a(x, u)= \begin{cases}1 & \text { if } x \notin\left(a_{1}, a_{2}\right), \\ \frac{u^{\prime}(x)}{v^{\prime}(x)} & \text { if } x \in\left(a_{1}, a_{2}\right) \text { and if } u \leq u(x), \\ \delta+(1-\delta) \frac{u^{\prime}(x)}{v^{\prime}(x)} & \text { if } x \in\left(a_{1}, a_{2}\right) \text { and if } u \geq v(x), \\ & \text { and if } u=\delta u\left(a_{1}, a_{2}\right)\end{cases}
$$

It is clear that $a$ defined that way is continuous in both variables $x, u$ (note that $u^{\prime}\left(a_{1}\right)=$ $\left.v^{\prime}\left(a_{2}\right), u^{\prime}\left(a_{2}\right)=v^{\prime}\left(a_{2}\right)\right)$. Moreover, (1.1) holds.

From this choice of $a$ one has obviously

$$
a(x, v) v^{\prime}=a(x, u) u^{\prime}=u^{\prime} \quad \text { on }\left(a_{1}, a_{2}\right)
$$

so that $u$ and $v$ are both solution to (3.1) with $f=-u^{\prime \prime}$.

Now, for $t$ small enough, there exists $x$ close to $a_{1}$ or $a_{2}$ such that $|u(x)-v(x)|=t$, then by (3.9),

$$
a(x, u(x))-a(x, v(x))=1-\frac{u^{\prime}(x)}{v^{\prime}(x)}=\frac{v^{\prime}(x)-u^{\prime}(x)}{v^{\prime}(x)} .
$$

But, in the neighbourhood of $a_{1}$ or $a_{2}$ one has (for instance for $a_{1}$, and by (3.6))

$$
(v-u)^{\prime}=\omega\left(\theta^{-1}\left(x-a_{1}\right)\right)=\omega(v-u)
$$

and thus,

$$
a(x, u(x))-a(x, v(x))=\frac{1}{v^{\prime}(x)} \omega(v(x)-u(x))=\frac{1}{v^{\prime}(x)} \omega(t) .
$$

So, for $t$ small $\omega_{a}(t) \geq C \omega(t)$ for some constant $C$, hence

$$
\int_{0^{+}} \frac{d s}{\omega_{a}(s)} \leq \frac{1}{C} \int_{0^{+}} \frac{d s}{\omega(s)}
$$

and (2.12) fails. Of course, one can show that (1.3) fails as well.

In the case where (3.4) holds, let us now prove that, for some constant $C$, one has also

$$
\omega_{a}(t) \leq C \omega(t) .
$$

For that, remark that if $P$ denotes the projection of $\mathbf{R}$ onto $[u(x), v(x)]$, i.e. if

$$
P(y)= \begin{cases}u(x) & \text { if } y \leq u(x), \\ y & \text { if } y \in[u(x), v(x)], \\ v(x) & \text { if } y \geq v(x),\end{cases}
$$

then, by definition of $a$ one has

$$
a(x, y)=a(x, P(y)) .
$$

So, if we prove that

$$
\left|a(x, z)-a\left(x, z^{\prime}\right)\right| \leq C \omega\left(\left|z-z^{\prime}\right|\right) \quad \forall z, z^{\prime} \in[u(x), v(x)], \text { a.e. } x \in \Omega
$$


we will have (3.12) since from (3.14) it will follow

$$
\begin{aligned}
|a(x, u)-a(x, v)| & =|a(x, P(u))-a(x, P(v))| \leq C \omega(|P(u)-P(v)|) \\
& \leq C \omega(|u-v|) \quad \forall u, v \in \mathbf{R}, \text { a.e. } x \in \Omega .
\end{aligned}
$$

To prove (3.15) consider for $\delta, \delta^{\prime} \in[0,1]$

$$
z=\delta u(x)+(1-\delta) v(x), \quad z^{\prime}=\delta^{\prime} u(x)+\left(1-\delta^{\prime}\right) v(x) .
$$

From (3.9) one has

$$
a(x, z)-a\left(x, z^{\prime}\right)=\left(\delta-\delta^{\prime}\right)\left[1-u^{\prime}(x) / v^{\prime}(x)\right]
$$

and

$$
z-z^{\prime}=\left(\delta-\delta^{\prime}\right)(u(x)-v(x)) .
$$

So, for $x$ outside of neighbourhoods of $a_{1}$ and $a_{2}$ one has

$$
\left|a(x, z)-a\left(x, z^{\prime}\right)\right|=\left|z-z^{\prime}\right|\left|1-u^{\prime}(x) / v^{\prime}(x)\right| /|u-v| \leq C\left|z-z^{\prime}\right| .
$$

Let us fix some $t_{0}>0$. Since by (3.4) the function $\omega(t) / t$ is nonincreasing, one has

$$
\omega(t) / t \geq \omega\left(t_{0}\right) / t_{0} \quad \forall t \leq t_{0}
$$

hence for some constant $C$

$$
\omega(t) \geq C t \quad \forall t \leq t_{0} .
$$

It then follows from (3.20) that

$$
\left|a(x, z)-a\left(x, z^{\prime}\right)\right| \leq C^{\prime} \omega\left(\left|z-z^{\prime}\right|\right) .
$$

$C^{\prime}$ depends of course on the neigbourhoods of $a_{1}, a_{2}$ considered. Note also that $a$ being bounded we need only to establish (3.23) for small values of $\left|z-z^{\prime}\right|$. Now, in the neighbourhood of $a_{1}$ or $a_{2}$, by (3.11), (3.18) one has

$$
\begin{aligned}
\left|a(x, z)-a\left(x, z^{\prime}\right)\right| & =\left|\delta-\delta^{\prime}\right|\left|\frac{v^{\prime}-u^{\prime}}{v^{\prime}}\right|=\left|\delta-\delta^{\prime}\right| \frac{\omega(v-u)}{v^{\prime}} \\
& =\frac{1}{v^{\prime}} \frac{\left|z-z^{\prime}\right|}{v-u} \omega(v-u) \\
& =\frac{1}{v^{\prime}} \omega\left(\left|z-z^{\prime}\right|\right) \frac{\left|z-z^{\prime}\right|}{\omega\left(\left|z-z^{\prime}\right|\right)} \frac{\omega(v-u)}{v-u} .
\end{aligned}
$$

Using (3.4) and the fact that $\left|z-z^{\prime}\right| \leq v-u$ we derive

$$
\left|a(x, z)-a\left(x, z^{\prime}\right)\right| \leq \frac{1}{v^{\prime}} \omega\left(\left|z-z^{\prime}\right|\right) .
$$

So, combining this with (3.23), we get for some constant $C$

$$
\left|a(x, z)-a\left(x, z^{\prime}\right)\right| \leq C \omega\left(\left|z-z^{\prime}\right|\right)
$$

and (3.12) follows.

In particular, when $\omega(t)=t^{\gamma}, \gamma \in(0,1)$ (note that for such an $\omega$ (3.3), (3.4) hold) (3.16) reads

$$
|a(x, u)-a(x, v)| \leq C|u-v|^{\gamma} \quad \forall u, v \in \mathbf{R}, \text { a.e. } x \in \Omega
$$

which is (3.2). This completes the proof of proposition 3.1. 
Remark 3.1. One can produce examples with more than two solutions by piling up different functions $v$.

Remark 3.2. When in (1.2) $g=0$, it is still possible to produce examples of nonuniqueness. For instance consider the construction we just made on $\left(a_{1}, a_{2}\right)=(-1,0)$ and with $0=A_{1}<A_{2}$. Then, symmetrise $u$ and $v$ on $(0,1)$. It is clear that we are producing that way a counterexample to uniqueness on $(-1,1)$ with homogeneous boundary data i.e. with $g=0$.

4. Concluding remarks. In fact Theorem 1 can also be rephrased into a comparison principle. More precisely we have:

THEOREM 1. Assume that for some positive constant $C$ one has

$$
|a(x, u)-a(y, u)| \leq C|x-y| \quad \forall u \in \mathbf{R}, \forall x, y \in \Omega
$$

or

$$
|a(x, u)-a(x, v)| \leq C|u-v| \quad \forall u, v \in \mathbf{R}, \text { a.e. } x \in \Omega .
$$

Let us denote by $u_{1}, u_{2}$ the solution to (1.2) corresponding respectively to the data $\left(f_{1}, g_{1}\right)$, $\left(f_{2}, g_{2}\right)$. Then if $f_{1} \leq f_{2}$ and $g_{1} \leq g_{2}$, one has $u_{1} \leq u_{2} .\left(f_{1} \leq f_{2}\right.$ is for instance taken in the $H^{-1}$ or in the measures sense).

Proof. In the case when (1.3) holds, (2.5) becomes

$$
-\frac{\partial}{\partial x_{i}}\left(\frac{\partial}{\partial x_{i}}\left(A\left(x, u_{1}\right)-A\left(x, u_{2}\right)\right)-\int_{u_{2}(x)}^{u_{1}(x)} \frac{\partial a(x, t)}{\partial x_{i}} d t\right) \leq 0 \quad \text { in } \Omega .
$$

So, one can establish (2.6) (with $u, v$ replaced by $u_{1}, u_{2}$ ) as in section 2 and conclude the same way that $u_{1} \leq u_{2}$.

In the case where $(1.4)$ holds, since $F_{\epsilon}\left(u_{1}-u_{2}\right) \in H_{0}^{1}(\Omega)$ and $F_{\epsilon}\left(u_{1}-u_{2}\right) \geq 0,(2.16)$ becomes

$$
\begin{aligned}
\int_{\Omega} a\left(x, u_{1}\right) \frac{\partial\left(u_{1}-u_{2}\right)}{\partial x_{i}} \cdot \frac{\partial}{\partial x_{i}} & F_{\epsilon}\left(u_{1}-u_{2}\right) d x \\
& \leq-\int_{\Omega}\left(a\left(x, u_{1}\right)-a\left(x, u_{2}\right)\right) \frac{\partial u_{2}}{\partial x_{i}} \cdot \frac{\partial}{\partial x_{i}} F_{\epsilon}\left(u_{1}-u_{2}\right) d x
\end{aligned}
$$

and the proof is the same.

The situation is quite different for the problem

$$
\left\{\begin{array}{l}
-\frac{\partial}{\partial x_{i}}\left(a(x, u) \frac{\partial u}{\partial x_{i}}\right)+\lambda(x) u=f \quad \text { in } \Omega, \\
u-g \in H_{0}^{1}(\Omega) .
\end{array}\right.
$$

where $\lambda(x)$ is a positive function. Here uniqueness can hold even when both (1.3), (1.4) fail. We refer the reader to $[\mathrm{A}],[\mathrm{AC}]$.

With a similar technique uniqueness and nonuniqueness results are also available for more general nonlinear problems as for instance variational inequalities associated to nonlinear operators of the type considered here (see $[\mathrm{CM}],[\mathrm{M}]$ ), or systems (see $[\mathrm{A}]$, $[\mathrm{CFM}])$. 
It is also possible to consider the parabolic analogue of our problem i.e.

$$
\begin{cases}\frac{\partial u}{\partial t}-\frac{\partial}{\partial x_{i}}\left(a(x, u) \frac{\partial u}{\partial x_{i}}\right)=f & \text { in } \Omega \times(0, T), \\ u-g \in H_{0}^{1}(\Omega) \quad t \in(0, T), & u(., 0)=u_{0} .\end{cases}
$$

In this situation uniqueness may also hold even if (1.3), (1.4) fail (see [Ar], [CR], [R]).

Acknowledgements. This work has been completed when the second author was visiting the University of Trento. We would like to thank the people of the mathematics department for their friendly hospitality.

\section{References}

[A] N. André, Thesis, University of Metz, in preparation.

[AC] N. André and M. Chipot, in preparation.

[Ar] M. Artola, Sur une classe de problèmes paraboliques quasilinéaires, Boll. Un. Mat. Ital. (6) 5-B (1986), 51-70.

[BKS] H. Brezis, D. Kinderlehrer et G. Stampacchia, Sur une nouvelle formulation du problème de l'écoulement à travers une digue, C. R. Acad. Sci. Paris Sér. A 287 (1978), 711-714.

[CC] J. Carrillo and M. Chipot, On nonlinear elliptic equations involving derivative of the nonlinearity, Proc. Roy. Soc. Edinburgh 100A (1985), 281-294.

[CM] M. Chipot and G. Michaille, Uniqueness results and monotonicity properties for the solution of some variational inequalities, Ann. Scuola Norm. Sup. Pisa (4) 16 (1989), 137-166.

[CFM] M. Chipot, A. Feggous and G. Michaille, Monotonicity properties for variational inequalities associated with nonlinear diagonal systems, in: Proceedings of the Nancy Meeting, 1988, Pitman Res. Notes 208, P. Bénilan, M. Chipot, L. C. Evans, M. Pierre (eds.), Longman, 1989, 255-266.

[CR] M. Chipot and J. F. Rodrigues, Comparison and stability of solutions to a class of quasilinear parabolic problems, Proc. Roy. Soc. Edinburgh 110A (1988), 275-285.

[GT] D. Gilbarg and N. S. Trudinger, Elliptic Partial Differential Equations of Second Order, Springer, Berlin, 1985.

[KS] D. Kinderlehrer and G. Stampacchia, An Introduction to Variational Inequalities, Academic Press, New York, 1980.

[M] G. Michaille, Thesis, University of Metz, 1988.

[R] J. F. Rodrigues, Strong solutions for quasilinear elliptic-parabolic problems with time dependent obstacles, in Proc. First European Conference on Elliptic and Parabolic Problems, Pont-à-Mousson, June 1991, Pitman Res. Notes in Math. 266, Longman, 70-82.

[T] N. S. Trudinger, On the comparison principle for quasilinear divergence structure equations, Arch. Rational Mech. Anal. 57 (1974), 128-133. 